\title{
Dynamic contrast-enhanced magnetic resonance imaging of osseous spine metastasis before and 1 hour after high-dose image-guided radiation therapy
}

\author{
${ }^{*}$ Eric Lis, MD, ${ }^{1}$ Atin Saha, MD, ${ }^{1}$ Kyung K. Peck, PhD, ${ }^{1,2}$ Joan Zatcky, NP, ${ }^{3}$ Michael J. Zelefsky, MD, ${ }^{3}$ \\ Yoshiya Yamada, MD, ${ }^{3}$ Andrei I. Holodny, MD, ${ }^{2}$ Mark H. Bilsky, MD, ${ }^{4}$ and Sasan Karimi, MD ${ }^{1}$ \\ Departments of ${ }^{1}$ Radiology, ${ }^{2}$ Medical Physics, ${ }^{3}$ Radiation Oncology, and ${ }^{4}$ Neurosurgery, Memorial Sloan Kettering Cancer \\ Center, New York, New York
}

\begin{abstract}
OBJECTIVE High-dose image-guided radiation therapy (HD IGRT) has been instrumental in mitigating some limitations of conventional RT. The recent emergence of dynamic contrast-enhanced (DCE) MRI to investigate tumor physiology can be used to verify the response of human tumors to HD IGRT. The purpose of this study was to evaluate the nearimmediate effects of HD IGRT on spine metastases through the use of DCE MRI perfusion studies.

METHODS Six patients with spine metastases from prostate, thyroid, and renal cell carcinoma who underwent HD IGRT were studied using DCE MRI prior to and 1 hour after HD IGRT. The DCE perfusion parameters plasma volume $\left(\mathrm{V}_{\mathrm{p}}\right)$ and vascular permeability $\left(\mathrm{K}_{\text {trans }}\right)$ were measured to assess the near-immediate and long-term tumor response. $\mathrm{A}$ Mann-Whitney U-test was performed to compare significant changes (at $p \leq 0.05$ ) in perfusion parameters before and after RT.
\end{abstract}

RESULTS The authors observed a precipitous drop in $V_{p}$ within 1 hour of HD IGRT, with a mean decrease of $65.2 \%$. A significant difference was found between $V_{p}$ values for before and 1 hour after $R T(p \leq 0.05)$. No significant change was seen in $V_{p}(p=0.31)$ and $K_{\text {trans }}(p=0.1)$ from 1 hour after RT to the first follow-up.

CONCLUSIONS The data suggest that there is an immediate effect of HD IGRT on the vascularity of spine metastases, as demonstrated by a precipitous decrease in $V_{p}$. The DCE MRI studies can detect such changes within 1 hour after RT, and findings are concordant with existing animal models.

https://thejns.org/doi/abs/10.3171/2016.9.FOCUS16378

KEY WORDS dynamic contrast-enhanced MRI; high-dose image-guided radiation therapy; spine tumor response

$\mathrm{T}$ HE third most common site of metastatic disease is the skeletal system, following lung and liver. Within the skeletal system, spine metastases represent the most common site of involvement. ${ }^{17}$ Significant pain resulting from destruction of the spinal column, leading to mechanical instability and neurological deficits due to spinal cord compression, are frequent sequelae of metastatic vertebral lesions. ${ }^{6,20}$ The treatment of metastatic cancer in the spine with radiation therapy (RT) has been well documented.

Conventional external-beam radiation (e.g., $30 \mathrm{~Gy}$ in 10 fractions) often results in poor local control rates for resistant tumors, such as renal cell carcinoma and mela- noma. Dose constraints using conventional techniques are calculated according to spinal cord tolerance. ${ }^{8,13}$ Furthermore, due to the anatomical location of the vertebral bodies, as well as organ and patient motion, conventional RT necessitates margins of 1-2 additional vertebral segments adjacent to the metastatic disease. The patient population with metastases is often critically ill, with multiple other comorbidities, which limits patients' ability to complete daily treatments. ${ }^{12}$

Techniques developed to deliver stereotactic radiosurgery have been instrumental in mitigating some of the limitations of conventional RT. ${ }^{3,9,15}$ Image-guided RT (IGRT) is valuable in sparing sensitive structures from high-dose

ABBREVIATIONS AIF = arterial input function; $A S M a s e=$ acid sphingomyelinase; $D C E=$ dynamic contrast-enhanced; $F A=$ flip angle; FOV = field of view; $H D I G R T=$ high-dose image-guided radiation therapy; $\mathrm{K}_{\text {trans }}=$ vascular permeability; $\mathrm{ROI}=$ region of interest; $\mathrm{V}_{\mathrm{p}}=$ plasma volume

SUBMITTED August 31, 2016. ACCEPTED September 21, 2016.

INCLUDE WHEN CITING DOI: 10.3171/2016.9.FOCUS16378.

* Drs. Lis and Saha contributed equally to this work. 
radiation-i.e., structures such as the spinal cord that are within millimeters of the metastatic lesion. ${ }^{19}$

Studies have illustrated tumor response to fractionated RT. ${ }^{10,11}$ The underlying mechanism of reduced tumor growth due to high single-dose radiation has been studied using experimental mouse tumor models. These studies have provided supporting evidence that the normal and tumor stem cell response to high single-dose radiation exposure ( $\geq 15 \mathrm{~Gy}$ ) is conditionally linked to the induction of microvascular endothelial apoptosis in the targeted tissue. Single-dose RT appears capable of engaging an early vascular component of the tumor response..$^{5,18}$

Dynamic contrast-enhanced (DCE) MRI can provide functional information in regard to tumor vascularity and hemodynamics through the calculation of perfusion parameters such as plasma volume $\left(\mathrm{V}_{\mathrm{p}}\right)$ and vascular permeability $\left(\mathrm{K}_{\text {trans }}\right)$. The use of DCE MRI in RT has been previously discussed. ${ }^{1}$ For DCE MRI parameters such as $\mathrm{K}_{\text {trans }}$, $V_{p}$, wash-in enhancement slope, and peak enhancement signal, the percentage change in areas of highest wash-in enhancement slope have been shown to be associated with the discrimination of hypervascular from hypovascular spine metastases. ${ }^{714}$ The recent emergence of DCE MRI to investigate tumor physiology has provided a tool that can be used to verify the human tumor physiology after high-dose IGRT (HD IGRT) compared with that of the animal models as well, with the potential to identify treatment response.

Studies have yet to illustrate the immediate posttreatment effects of HD IGRT. Our goal was to evaluate the near-immediate effects on $\mathrm{V}_{\mathrm{p}}$ and $\mathrm{K}_{\text {trans }}$, the DCE MRI perfusion parameters of spine metastases that have undergone HD IGRT.

\section{Methods}

An institutional review board waiver of authorization was granted prior to the study. Six patients were treated for 8 metastatic spine tumors, including 5 lumbar and 3 thoracic. All patients were male, with a mean age of 60 years. The primary site of spine metastases included 4 prostate and 1 each of renal cell and thyroid (Table 1).

All patients underwent standard MRI both before and after HD IGRT (1.5-T GE scanner using an 8-channel cervical-thoracic-lumbar surface spinal coil). Images included sagittal T1-weighted (field of view [FOV] 32-36 cm, slice thickness $3 \mathrm{~mm}$, TR 400-650 msec, flip angle [FA] $90^{\circ}$ ); sagittal T2-weighted (FOV 32-36 cm, slice thickness $3 \mathrm{~mm}$, TR 3500-4000 msec, FA $90^{\circ}$ ); and sagittal STIR (FOV 32-36 cm, slice thickness $3 \mathrm{~mm}$, TR 3500-6000 msec, FA $90^{\circ}$ ). For MRI scans obtained before and after dynamic contrast enhancement, a T1-weighted 3D fast spoiled gradient-recalled acquisition sequence with the following parameters was used: TR/TE 3.6/1.1 msec, slice thickness $10 \mathrm{~mm}$, FA $30^{\circ}$, FOV $34 \mathrm{~cm}$, matrix size $256 \times$ 128 in the sagittal plane, number of phases 80 . Gd-DTPA at a dose of $0.1 \mathrm{mmol} / \mathrm{kg}$ of body weight was administered at a rate of $2.5 \mathrm{ml} / \mathrm{sec}$ by power injector.

Data processing and analysis was performed using dynamic image processing software (NordicIce-NeuroLab) and Matlab (Mathworks). Preprocessing steps include background noise removal and spatial and temporal smoothing to reduce high-frequency noise. Automatic detection of the arterial input function (AIF) from the aorta was applied. The AIF was individually calculated in each image acquisition in every patient. Appropriate shape of the AIF curve was visually confirmed before processing steps continued. All regions of interest (ROIs) around the metastatic lesions were placed by a single neuroradiologist (S.K.) in the solid part of the tumor, with careful consideration to avoid venous structures, hemangiomas, disc spaces, cortical bone, and spondylotic changes. Anatomical images that matched the DCE MR images were used in ROI placements. Voxel-by-voxel estimates of quantitative perfusion parameters, including $\mathrm{K}_{\text {trans }}$ and $\mathrm{V}_{\mathrm{p}}$, were determined using a Toft's pharmacokinetic model analysis. ${ }^{16}$

All metastases underwent HD IGRT in one of two dose strategies: 24-Gy single fraction (7 tumors) or $27 \mathrm{~Gy}$ in 3 fractions (1 tumor). Repeat DCE MRI was performed within 1 hour of completion of the RT and then at subsequent follow-up imaging. To assess the perfusion parameters as predictors of treatment response, $\mathrm{V}_{\mathrm{p}}$ and $\mathrm{K}_{\text {trans }}$ of the metastases were calculated before and after RT. These parameters were normalized as a ratio to adjacent nonirradiated marrow and compared before and after RT.

\section{Data Analysis: $\mathrm{V}_{\mathrm{p}}$ and $\mathrm{K}_{\text {trans }}$ Values 1 Hour After RT}

A Mann-Whitney U-test was used to quantify the significance of a drop in $\mathrm{V}_{\mathrm{p}}$ and $\mathrm{K}_{\text {trans }} 1$ hour after HD IGRT treatment. The preoperative $\mathrm{V}_{\mathrm{p}}$ and $\mathrm{K}_{\text {trans }}$ values were compared with the 1-hour posttreatment $\mathrm{V}_{\mathrm{p}}$ and $\mathrm{K}_{\text {trans }}$ values,

TABLE 1. Demographic and treatment parameters in 6 patients with spinal metastases

\begin{tabular}{|c|c|c|c|c|c|c|c|}
\hline Case No.* & Age (yrs) & Sex & Pathology & Tx Region & Tx Dose† & Tx Dates & Follow-Up MRI \\
\hline 1 & 58 & M & Thyroid carcinoma & L-4‡ & $24 \mathrm{~Gy}$ & $3 / 12 / 2010$ & $1 \mathrm{hr}, 61$ days, 586 days \\
\hline $2 A, B$ & 56 & M & Prostate adenocarcinoma & L-2, L-5 & $24 \mathrm{~Gy}$ & $10 / 8 / 2009$ & $1 \mathrm{hr}, 51$ days, 839 days \\
\hline $3 A, B$ & 52 & M & Prostate adenocarcinoma & $\mathrm{T}-5, \mathrm{~T}-11$ & $24 \mathrm{~Gy}$ & $11 / 10 / 2009$ & $1 \mathrm{hr}, 56$ days \\
\hline 4 & 76 & M & Prostate adenocarcinoma & $\mathrm{T}-10$ & $24 \mathrm{~Gy}$ & $1 / 28 / 2010$ & $1 \mathrm{hr}, 57$ days, 194 days \\
\hline 5 & 59 & M & Prostate adenocarcinoma & L-3 & $24 \mathrm{~Gy}$ & $9 / 24 / 2009$ & $1 \mathrm{hr}, 504$ days \\
\hline 6 & 57 & M & Renal cell carcinoma & L-4 & 9 Gy (× 3 fractions) & $3 / 12 / 2012$ & $1 \mathrm{hr}, 73$ days \\
\hline
\end{tabular}

Tx = treatment.

* $A$ and $B$ denote multiple metastases sites for the same patient.

$\dagger$ For all tumors treated to $24 \mathrm{~Gy}$, the doses were delivered in a single fraction.

$\ddagger$ Another metastasis, to L-5, occurred later. 
respectively. Furthermore, the 1-hour posttreatment $V_{p}$ and $\mathrm{K}_{\text {trans }}$ values were also compared with the first followup $\mathrm{V}_{\mathrm{p}}$ and $\mathrm{K}_{\text {trans }}$ values. A 1-tailed Mann-Whitney U-test at a significance level of $\mathrm{p} \leq 0.05$ was conducted.

\section{Results}

One hour after HD IGRT, the DCE MRI studies showed a significant drop in the $\mathrm{V}_{\mathrm{p}}$ for all 6 metastases $(\mathrm{p}=0.03)$, indicating a lower vascular $\mathrm{V}_{\mathrm{p}}$ in the tumors after treatment. The $\mathrm{K}_{\text {trans }}$ remained stable for 3 cases and showed a decrease for the remaining 3 cases $(p=0.06)$. There was a change in $\mathrm{V}_{\mathrm{p}}(\mathrm{p}=0.31)$ and $\mathrm{K}_{\text {trans }}(\mathrm{p}=0.1)$ between 1 hour after RT and the first follow-up, but it was not a statistically significant change, which is consistent with the failure of tumor growth verified on imaging.

\section{Structural Image}

Admission T1-weighted MRI scans were obtained to visualize the location of the metastases prior to HD IGRT (Fig. 1).

\section{The T1-Weighted DCE Perfusion Maps}

The T1-weighted DCE MRI perfusion maps illustrate the hemodynamic properties of the spine metastases (Fig. 2). Pretreatment tumor shows an increased hyperintensity for both $\mathrm{V}_{\mathrm{p}}$ and $\mathrm{K}_{\text {trans }}$, which is in stark contrast to the reduced intensity levels shown for $V_{p}$ and $K_{\text {trans }}$ at 1 hour posttreatment as well as at subsequent posttreatment follow-ups. The reduction in intensity of the perfusion parameters correlates with the decrease in vascularity of the tumor, indicating a response.

\section{The $V_{p}$ and $K_{\text {trans }}$ Values}

The $\mathrm{V}_{\mathrm{p}}$ illustrates a precipitous drop 1 hour after HD IGRT, indicating decreased perfusion of the spine metastasis (Fig. 3). There was an average drop of $65.2 \%$ in $\mathrm{V}_{\mathrm{p}} 1$ hour posttreatment among all 6 patients. Subsequent follow-up scans showed continued decrease in perfusion; however, the $\mathrm{V}_{\mathrm{p}}$ decline was far less on subsequent followup studies. The median preoperative $\mathrm{V}_{\mathrm{p}}$ was 15.14 , whereas the median 1-hour posttreatment $\mathrm{V}_{\mathrm{p}}$ was 3.94. Similar to the $\mathrm{V}_{\mathrm{p}}$ trends, the permeability factor (or $\mathrm{K}_{\text {trans }}$ ) of the DCE MRI illustrated a slight drop at 1 hour after RT (median 2.3) from the preoperative value (median 4.84). In concurrent follow-ups, the permeability factor remained stable.

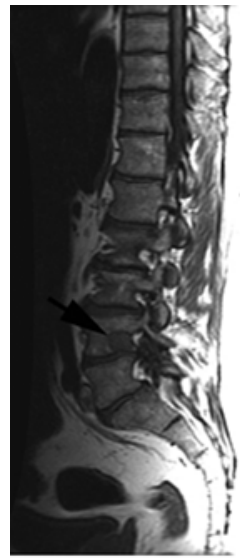

Patient 1

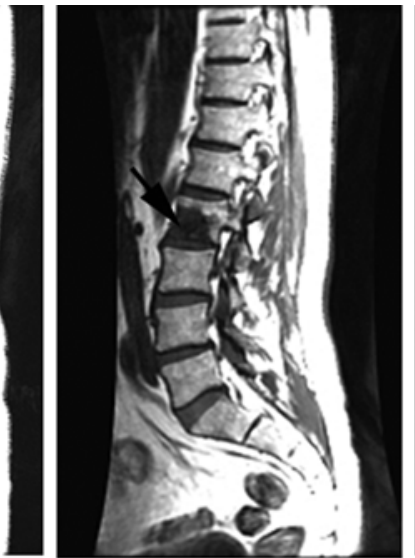

Patient 2

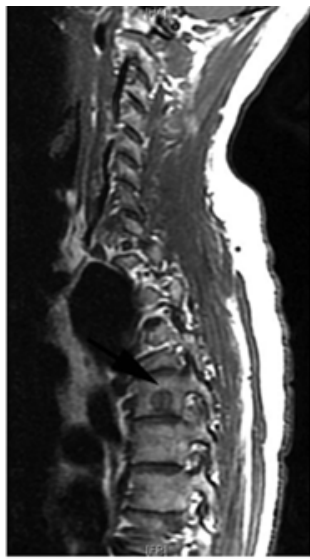

Patient 3A

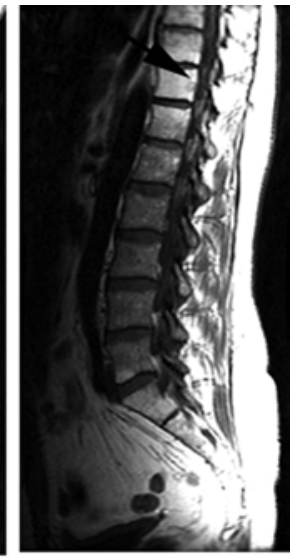

Patient 3B

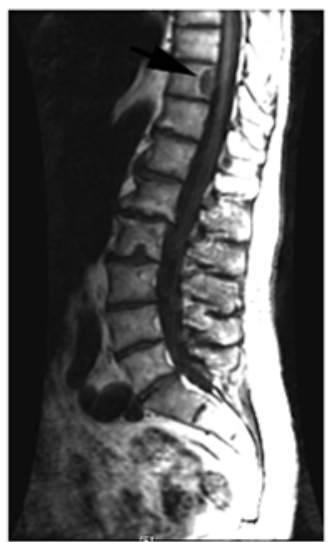

Patient 4

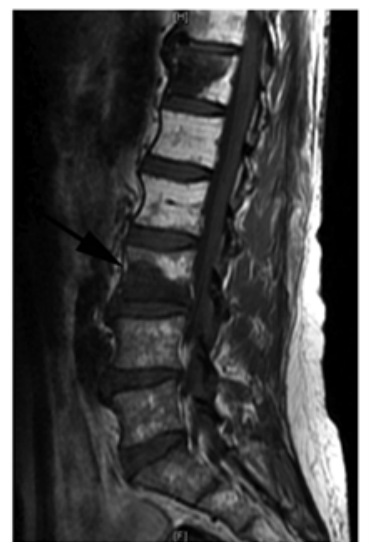

Patient 5

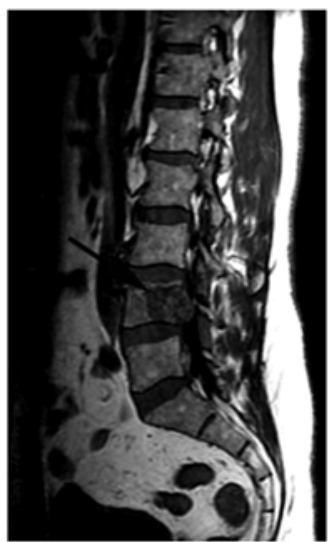

Patient 6

FIG. 1. Sagittal T1-weighted MRI study illustrates the structural anatomy of the spine for all 6 patients prior to treatment with $H D$ IGRT. The patient in Case 1 shows a metastatic lesion at L-4; the patient in Case 2 at L-2 (lesion A-lesion B not shown); the patient in Case 3 at T-5 (lesion A) and T-11 (lesion B); the patient in Case 4 at T-10; the patient in Case 5 at L-3; and the patient in Case 6 at L-4. The patient labeling is in concordance with Table 1. 


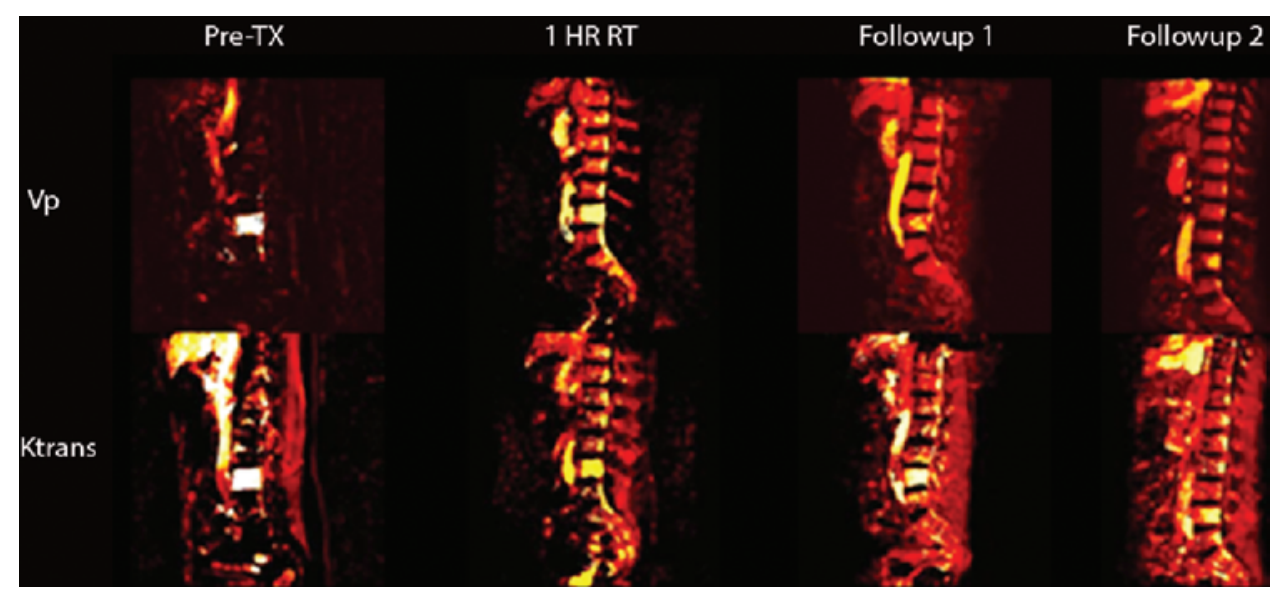

FIG. 2. Sagittal T1-weighted DCE MRI perfusion maps for the parameters $V_{p}$ and $K_{\text {trans }}$ at times pre-RT, 1 hour post-RT, and subsequent follow-ups. The images were obtained in the patient in Case 6 and show the perfusion of spine metastasis to L-4 from renal cell carcinoma. The $V_{p}$ decreased from 36.7 preoperatively to 1.85 at 1 hour post-RT (a $95 \%$ decrease). The $\mathrm{K}_{\text {trans }}$ map shows a similar decrease from 14 preoperatively to 4 at 1 hour post-RT (a $71.4 \%$ decrease). $\mathrm{TX}=$ treatment.

\section{Correlation of Tumor Progression and Perfusion}

At follow-up visit, imaging indicated a new spine metastasis to L-5 for the patient in Case 1, who had previously undergone single-fraction HD IGRT to treat spine metastases in L-4 (Fig. 4). Perfusion imaging corroborates this finding, with decreased perfusion seen in the treated L-4
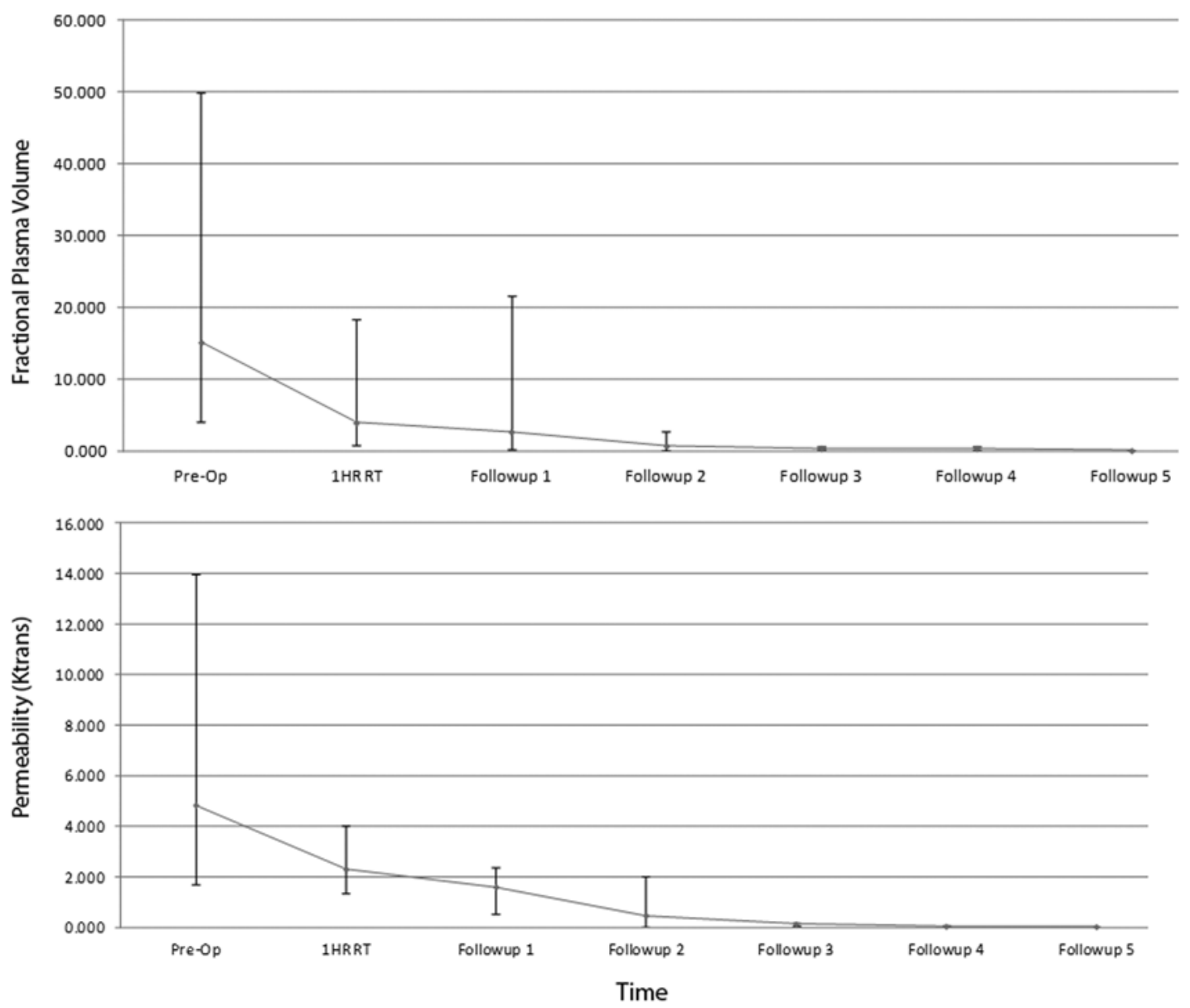

FIG. 3. A line plot illustrating the median $V_{p}$ and $K_{\text {trans }}$ at various times, including before RT, 1 hour after RT, and at different followup sessions. The bars and whiskers illustrate the range in $V_{p}$ and $K_{\text {trans }}$ values recorded from the patients' T1-weighted DCE MRI scans. The $\mathrm{V}_{\mathrm{p}}$ ranged from 4.1 to 50 before RT and from 0.78 to 18.3 at 1 hour after RT. The $\mathrm{K}_{\text {trans }}$ ranged from 1.7 to 14 before RT and from 1.35 to 4 at 1 hour after RT. 


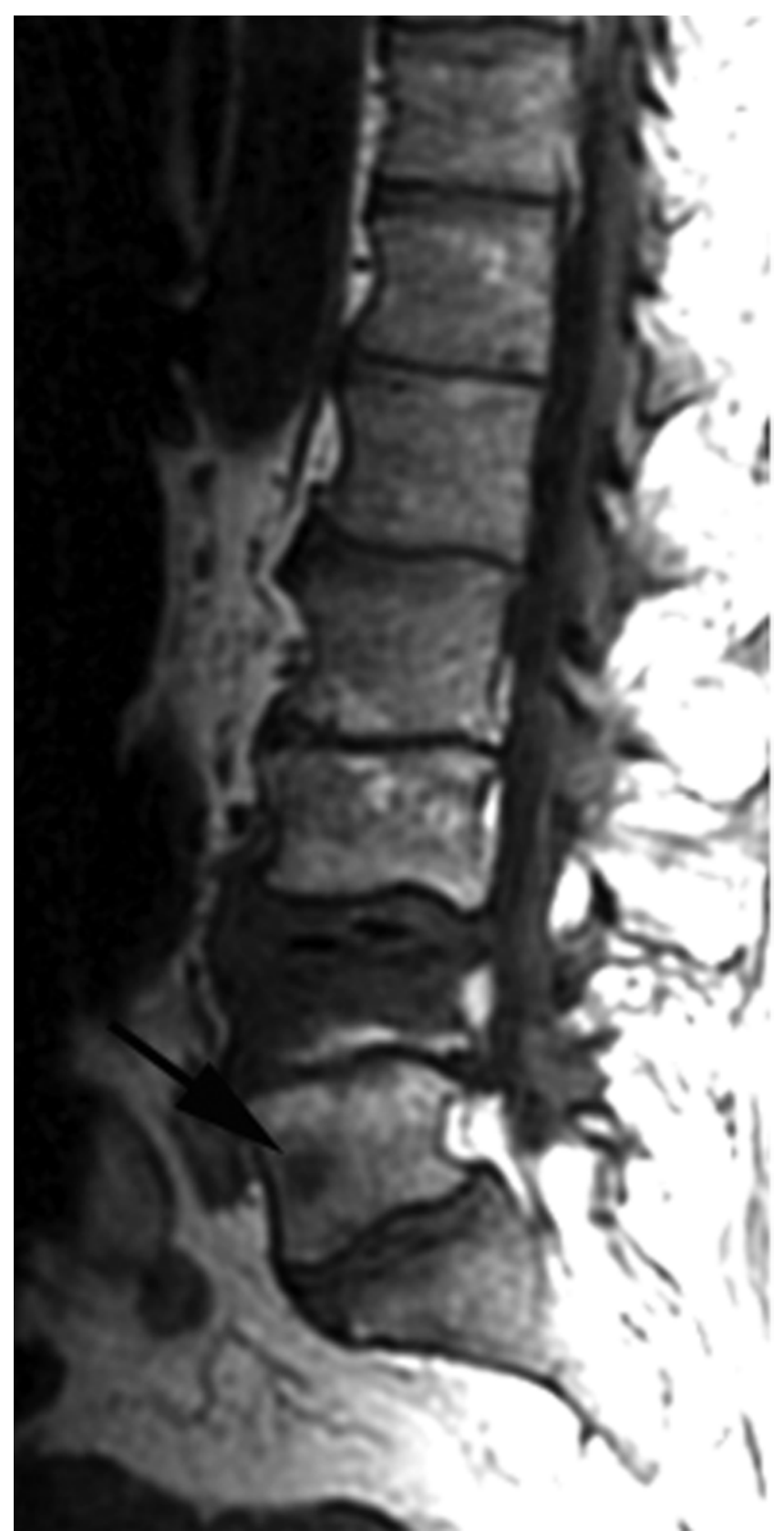

FIG. 4. Case 1. Sagittal T1-weighted structural image showing a new metastatic lesion in L-5 (arrow).

metastases and hyperperfusion for the newly identified L-5 spine metastases (Fig. 5). Perfusion imaging shows findings concurrent with conventional MRI studies.

\section{Discussion}

The goal of this study was to evaluate the near-immediate effects of HD IGRT on spine metastases through the use of DCE MRI perfusion studies. It has been shown that blood perfusion changes, particularly illustrated by the T1-weighted DCE MRI vascular parameter, $\mathrm{V}_{\mathrm{p}}$, reflect tumor response to RT in spinal bone metastases. ${ }^{2}$ The $V_{p}$ is a measure of intravascular volume, and a decline in $\mathrm{V}_{\mathrm{p}}$ after RT probably represents the reduction in vascularity of the successfully treated lesion. The results of our study demonstrate that DCE perfusion imaging correlates with treatment response, as indicated by long-term followup and clinical assessments. This correlates with animal studies as well, in which it was shown that a single dose of radiation (15-20 Gy) to mice with MCA129 fibrosarcoma and B16 melanoma resulted in a rapid wave of endothelial apoptosis, illustrating anatomical evidence of tissue damage 1-6 hours after RT. ${ }^{4}$ Apoptotic endothelial cell death involves complex biochemical changes and is mediated by the acid sphingomyelinase (ASMase) pathway. Radiation induces the translocation of the secretory form of ASMase from cytosol into glycosphingolipid contained in the outer leaflet of the plasma membrane, which then hydrolyzes the sphingomyelin to generate the proapoptotic second messenger ceramide. Endothelial cells are enriched with secretory ASMase at a level 20 times that of any other cell in the body, and as a result are sensitive to radiation-induced apoptosis.

The DCE MRI technique demonstrates functional information on tumor vascularity and hemodynamics. A quantitative assessment of vascular features can be achieved by applying a pharmacokinetic model of contrast uptake to the calculated signal intensity changes over time. DCE MRI has the capacity to be a sensitive technique that can detect hemodynamic changes in spine metastases treated with HD IGRT within 1 hour posttreatment. Our clinical findings show concordance with those of animal models, and we anticipate that a similar process is occurring in patients with metastatic disease to the spine after IGRT. The sharp decrease in $\mathrm{V}_{\mathrm{p}}$ (mean $37.8 \%$ ) from pretreatment to less than 1 hour posttreatment is in concordance with clinical findings. Longterm follow-up imaging demonstrated a failure of tumor progression for all 6 patients who underwent HD IGRT, indicating successful treatment of the spine metastases. These results also illustrate the advantage of DCE MRI over conventional imaging, which showed no change in appearance of metastases for months after treatment. Logistically, the study provided challenges in that patients were required to undergo RT and then to be transported to the imaging unit within 1 hour after RT. The patients were also required to have long-term follow-up clinically and radiographically.

The sample size was limited in the present study to include only patients who received HD IGRT and in whom a series of DCE MRI studies were obtained over time. To apply a pharmacokinetic model for quantitative analysis, both the precontrast T1 value and AIF must be accurately measured. In the design of DCE MRI acquisition, competing demands for high spatial resolution, coverage, and signal-to-noise ratios often result in inadequate temporal resolution for reliable measurement of the AIF. This is because a low sampling rate due to low temporal resolution can affect the time course of AIF and initial contrast agent wash-in. Saturation effects and missampling can affect the AIF time course and initial contrast bolus, and dispersion of the AIF before it reaches the ROI can also affect DCE MRI quantification. A standard method of analyzing perfusion data by selecting an ROI was used. This method 


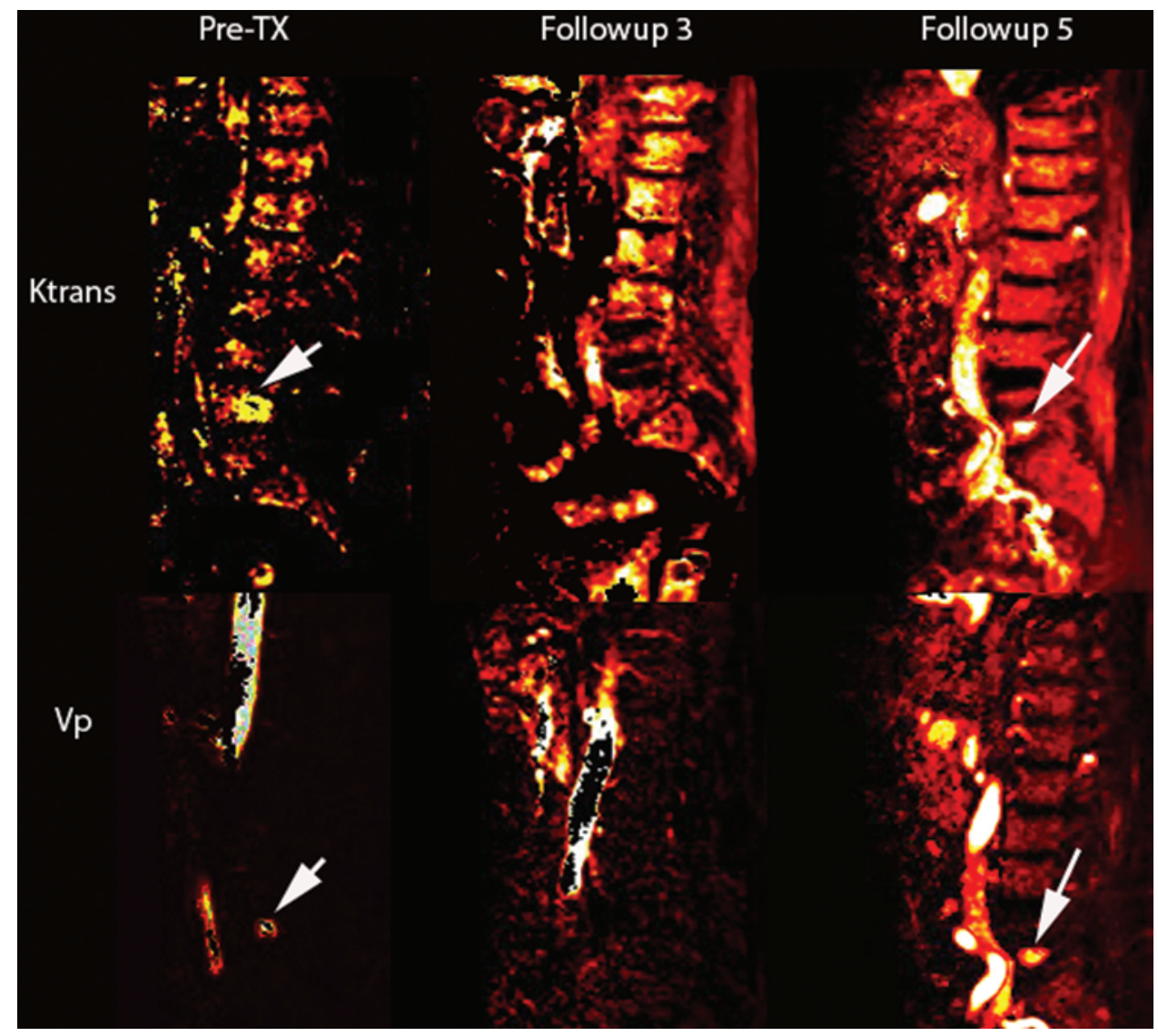

FIG. 5. Case 1. Sagittal T1-weighted DCE MRI perfusion maps for the parameters $V_{p}$ and $K_{\text {trans }}$ before $R T$, and at follow-up visits 3 and 5 after RT. Follow-up 3 illustrates a hypointensity at L-5, whereas follow-up 5 shows a hyperintensity with $V_{p}$ values of 5.4 and $\mathrm{K}_{\text {trans }}$ of 1.86. The $\mathrm{V}_{p}$ color map is consistent with the clinical finding of new metastases to $L-5$. The arrows designate untreated $L-4$ metastasis prior to radiation therapy.

has been shown to be reproducible, although it remains a subjective operator-dependent technique, introducing an inevitable factor of interobserver and intraobserver variability.

\section{Conclusions}

We successfully performed serial DCE MRI studies at constant and frequent intervals in patients with spine metastases who had undergone HD IGRT. Our preliminary results suggest that there is an immediate effect of HD IGRT on spine metastases in terms of the vascularity of the lesion, as demonstrated by a precipitous drop in $\mathrm{V}_{\mathrm{p}}$, which is a representation of tumor vascularity. We show that DCE MRI can detect such changes less than 1 hour after RT, and this finding is concordant with existing animal models. Changes in blood perfusion may reflect tumor responses to RT in bone marrow. Our long-term follow-up imaging studies confirm that indeed the changes in $\mathrm{V}_{\mathrm{p}}$ can be appreciated immediately after RT.

\section{Acknowledgments}

This work was supported in part by NIH/NCI core grant P30 CA008748.

\section{References}

1. Cao Y: The promise of dynamic contrast-enhanced imaging in radiation therapy. Semin Radiat Oncol 21:147-156, 2011

2. Chu S, Karimi S, Peck KK, Yamada Y, Lis E, Lyo J, et al: Measurement of blood perfusion in spinal metastases with dynamic contrast-enhanced magnetic resonance imaging: evaluation of tumor response to radiation therapy. Spine (Phila Pa 1976) 38:E1418-E1424, 2013

3. Cosgrove VP, Jahn U, Pfaender M, Bauer S, Budach V, Wurm RE: Commissioning of a micro multi-leaf collimator and planning system for stereotactic radiosurgery. Radiother Oncol 50:325-336, 1999

4. Fuks Z, Kolesnick R: Engaging the vascular component of the tumor response. Cancer Cell 8:89-91, 2005

5. Garcia-Barros M, Paris F, Cordon-Cardo C, Lyden D, Rafii S, Haimovitz-Friedman A, et al: Tumor response to radiotherapy regulated by endothelial cell apoptosis. Science 300:1155-1159, 2003

6. Gerszten PC, Germanwala A, Burton SA, Welch WC, Ozhasoglu C, Vogel WJ: Combination kyphoplasty and spinal radiosurgery: a new treatment paradigm for pathological fractures. J Neurosurg Spine 3:296-301, 2005

7. Khadem NR, Karimi S, Peck KK, Yamada Y, Lis E, Lyo J, et al: Characterizing hypervascular and hypovascular metastases and normal bone marrow of the spine using dynamic contrast-enhanced MR imaging. AJNR Am J Neuroradiol 33:2178-2185, 2012

8. Kopelson G, Linggood RM, Kleinman GM, Doucette J, 
Wang CC: Management of intramedullary spinal cord tumors. Radiology 135:473-479, 1980

9. Marks LB, Sherouse GW, Das S, Bentel GC, Spencer DP, Turner D: Conformal radiation therapy with fixed shaped coplanar or noncoplanar radiation beam bouquets: a possible alternative to radiosurgery. Int J Radiat Oncol Biol Phys 33:1209-1219, 1995

10. Moeller BJ, Cao Y, Li CY, Dewhirst MW: Radiation activates HIF-1 to regulate vascular radiosensitivity in tumors: role of reoxygenation, free radicals, and stress granules. Cancer Cell 5:429-441, 2004

11. Moeller BJ, Dreher MR, Rabbani ZN, Schroeder T, Cao Y, Li CY, et al: Pleiotropic effects of HIF-1 blockade on tumor radiosensitivity. Cancer Cell 8:99-110, 2005

12. Ryu S, Fang Yin F, Rock J, Zhu J, Chu A, Kagan E, et al: Image-guided and intensity-modulated radiosurgery for patients with spinal metastasis. Cancer 97:2013-2018, 2003

13. Ryu SI, Chang SD, Kim DH, Murphy MJ, Le QT, Martin DP, et al: Image-guided hypo-fractionated stereotactic radiosurgery to spinal lesions. Neurosurgery 49:838-846, 2001

14. Saha A, Peck KK, Lis E, Holodny AI, Yamada Y, Karimi $\mathrm{S}$ : Magnetic resonance perfusion characteristics of hypervascular renal and hypovascular prostate spinal metastases: clinical utilities and implications. Spine (Phila Pa 1976) 39:E1433-E1440, 2014

15. Shiu AS, Kooy HM, Ewton JR, Tung SS, Wong J, Antes K, et al: Comparison of miniature multileaf collimation (MMLC) with circular collimation for stereotactic treatment. Int J Radiat Oncol Biol Phys 37:679-688, 1997

16. Tofts PS, Brix G, Buckley DL, Evelhoch JL, Henderson E, Knopp MV, et al: Estimating kinetic parameters from dynamic contrast-enhanced $\mathrm{T}_{1}$-weighted MRI of a diffusable tracer: standardized quantities and symbols. J Magn Reson Imaging 10:223-232, 1999

17. Togawa D, Lewandrowski KU: The pathophysiology of spinal metastases, in McLain RF, Lewandrowski KU, Markman M, et al (eds): Cancer in the Spine. Totowa, NJ: Human Press, 2006, pp 17-23

18. Yamada Y, Bilsky MH, Lovelock DM, Venkatraman ES, Toner S, Johnson J, et al: High-dose, single-fraction image- guided intensity-modulated radiotherapy for metastatic spinal lesions. Int J Radiat Oncol Biol Phys 71:484-490, 2008

19. Yamada Y, Lovelock DM, Bilsky MH: A review of imageguided intensity-modulated radiotherapy for spinal tumors. Neurosurgery 61:226-235, 2007

20. Yin FF, Ryu S, Ajlouni M, Yan H, Jin JY, Lee SW, et al: Image-guided procedures for intensity-modulated spinal radiosurgery. Technical note. J Neurosurg 101 (Suppl 3):419_ 424,2004

\section{Disclosures}

Dr. Lis is a consultant for Medtronic. Dr. Yamada is a consultant for Varian Medical Systems, and he serves on the medical advisory board for the Chordoma Foundation.

\section{Author Contributions}

Conception and design: Karimi, Lis, Peck, Yamada, Holodny. Acquisition of data: Karimi, Lis, Saha, Peck. Analysis and interpretation of data: Karimi, Lis, Saha, Peck, Yamada. Drafting the article: Karimi, Lis, Saha, Yamada, Bilsky. Critically revising the article: Karimi, Lis, Peck, Yamada, Bilsky. Reviewed submitted version of manuscript: Karimi, Lis, Yamada, Bilsky. Approved the final version of the manuscript on behalf of all authors: Karimi. Administrative/technical/material support: Zatcky, Zelefsky, Holodny.

\section{Supplemental Information}

Previous Presentations

A portion of the data in this manuscript was presented at Proceedings of the American Society of Neuroradiology 50th Annual Meeting \& the Foundation of the ASNR Symposium, held in New York, New York, on April 21-26, 2012.

\section{Correspondence}

Sasan Karimi, Department of Radiology, Memorial Sloan Kettering Cancer Center, 1275 York Ave., New York, NY 10065. email: karimis@mskcc.org. 